\title{
Diminished Cortical Thickness Is Associated with Impulsive Choice in Adolescence
}

\author{
Marieta Pehlivanova, ${ }^{1}$ Daniel H. Wolf, 2 Aristeidis Sotiras, ${ }^{3}$-Antonia N. Kaczkurkin, ${ }^{2}$ T)Tyler M. Moore, ${ }^{2}$

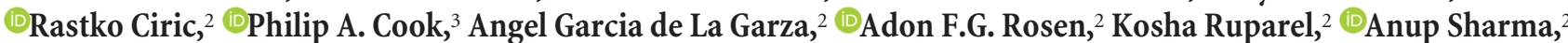 \\ @Russell T. Shinohara, ${ }^{4}$ David R. Roalf, ${ }^{2}$ Ruben C. Gur, ${ }^{2,3}$ Christos Davatzikos, ${ }^{3}$ Raquel E. Gur, ${ }^{2,3}$ Joseph W. Kable, ${ }^{1 *}$ \\ and ${ }^{-T h e o d o r e ~ D . ~ S a t t e r t h w a i t e ~}{ }^{2 *}$ \\ ${ }^{1}$ Departments of Psychology, ${ }^{2}$ Psychiatry and ${ }^{3}$ Radiology, Perelman School of Medicine, and ${ }^{4}$ Biostatistics, Epidemiology, and Informatics, University of \\ Pennsylvania, Philadelphia, Pennsylvania 19104
}

Adolescence is characterized by both maturation of brain structure and increased risk of negative outcomes from behaviors associated with impulsive decision-making. One important index of impulsive choice is delay discounting (DD), which measures the tendency to prefer smaller rewards available soon over larger rewards delivered after a delay. However, it remains largely unknown how individual differences in structural brain development may be associated with impulsive choice during adolescence. Leveraging a unique large sample of 427 human youths ( 208 males and 219 females) imaged as part of the Philadelphia Neurodevelopmental Cohort, we examined associations between delay discounting and cortical thickness within structural covariance networks. These structural networks were derived using non-negative matrix factorization, an advanced multivariate technique for dimensionality reduction, and analyzed using generalized additive models with penalized splines to capture both linear and nonlinear developmental effects. We found that impulsive choice, as measured by greater discounting, was most strongly associated with diminished cortical thickness in structural brain networks that encompassed the ventromedial prefrontal cortex, orbitofrontal cortex, temporal pole, and temporoparietal junction. Furthermore, structural brain networks predicted DD above and beyond cognitive performance. Together, these results suggest that reduced cortical thickness in regions known to be involved in value-based decision-making is a marker of impulsive choice during the critical period of adolescence.

Key words: adolescence; delay discounting; development; impulsivity; networks; neuroimaging

\section{Significance Statement}

Risky behaviors during adolescence, such as initiation of substance use or reckless driving, are a major source of morbidity and mortality. In this study, we present evidence from a large sample of youths that diminished cortical thickness in specific structural brain networks is associated with impulsive choice. Notably, the strongest association between impulsive choice and brain structure was seen in regions implicated in value-based decision-making; namely, the ventromedial prefrontal and orbitofrontal cortices. Moving forward, such neuroanatomical markers of impulsivity may aid in the development of personalized interventions targeted to reduce risk of negative outcomes resulting from impulsivity during adolescence.

\section{Introduction}

Adolescence is marked by an increased vulnerability to risky behaviors, such as tobacco, alcohol, and drug use, reckless driving,

Received Aug. 2, 2017; revised Jan. 8, 2018; accepted Jan. 11, 2018.

Author contributions: M.P., D.H.W., R.C.G., R.E.G., J.W.K., and T.D.S. designed research; M.P., A.N.K., A. Sharma, R.C.G., C.D., R.E.G., and T.D.S. performed research; A. Sotiras, A.N.K., T.M.M., R.C., P.A.C., A.G.d.L.G., A.F.G.R., K.R., R.T.S., D.R.R., C.D., J.W.K., and T.D.S. contributed unpublished reagents/analytic tools; M.P., A. Sotiras, T.M.M., R.C., P.A.C., A.G.d.L.G., A.F.G.R., K.R., R.T.S., and D.R.R. analyzed data; M.P., D.H.W., A. Sotiras, A.N.K., T.M.M., R.C.,P.A.C., A.G.d.L.G., A.F.G.R., K.R., A. Sharma, R.T.S., D.R.R., R.C.G., C.D., R.E.G., J.W.K., and T.D.S. wrote the paper.

This work was supported by Grants from the National Institute of Mental Health R01MH107703 (T.D.S.); NIMH RC2 Grants MH089983 and MH089924 (R.E.G.) for PNC; MH098899 and DA029149 (J.W.K.), K01MH102609 (D.R.R.), R01MH101111 (D.H.W.), R01-AG014971 and RF1-AG054409 (C.D., A.S.), P50MH096891 (R.E.G.), R01NS085211 (R.T.S.), and R01MH107235 (R.C.G.); the Dowshen Program for Neuroscience, and the Lifespan Brain Institute at the Children's Hospital of Philadelphia and Penn Medicine; Seed Grant support by the Center for Biomedical Computing and unprotected sex (Eaton et al., 2012). During this vulnerable period, the brain undergoes dramatic structural changes (Giedd et al., 1999; Sowell et al., 2004). Some evidence suggests that risk and Image Analysis (CBICA) for developing statistical analyses (R.T.S., T.D.S.) and non-negative matrix factorization software (A.S., T.D.S.); and Grants P50 AG05681, P01 AG03991, R01 AG021910, P20 MH071616, and U24 RRO21382 for images from the OASIS project. We thank the acquisition and recruitment team including Karthik Prabhakaran and Jeff Valdez, Chad Jackson for data management and systems support, and Monica Calkins for phenotyping expertise.

The authors declare no competing financial interests.

*J.W.K. and T.D.S. contributed equally as last authors.

Correspondence should be addressed to Dr. Theodore D. Satterthwaite, Gates Building, 10th Floor, Hospital of the University of Pennsylvania, 34th and Spruce Street, Philadelphia, PA 19104. E-mail: sattertt@upenn.edu. DOI:10.1523/JNEUROSCI.2200-17.2018

Copyright $@ 2018$ the authors $\quad 0270-6474 / 18 / 382471-11 \$ 15.00 / 0$ 
during adolescence is associated with differential maturation of brain regions related to reward processing (such as the orbitofrontal cortex and ventral striatum) and those necessary for cognitive control [such as the dorsolateral prefrontal cortex (dlPFC); Casey et al., 2008; Van Leijenhorst et al., 2010]. One of the most commonly used indices of impulsive choice is delay discounting (DD), a behavioral measure of impulsivity where one chooses between a smaller reward delivered sooner, and a larger reward with a longer delay (Kirby and Maraković, 1995; Peters and Büchel, 2011; Kable, 2013). Delay discounting engages regions known to mature at different rates in adolescence, including dlPFC (Peters and Büchel, 2011), orbitofrontal cortex, and ventral striatum (Kable and Glimcher, 2007; Bartra et al., 2013). Increased DD has been proposed as a framework for understanding substance abuse and other risky decisions as reflecting impulsive choices of immediate rewards (Bickel et al., 2007). Indeed, studies of adolescents show that higher impulsivity, as indexed by higher discounting, is associated with increased smoking frequency (Reynolds, 2004), greater alcohol consumption (Field et al., 2007), and predicts longitudinal increase in both smoking (Audrain-McGovern et al., 2009) and alcohol use (Fernie et al., 2013).

At present, it remains relatively unknown how individual differences in structural brain development may relate to DD in adolescents. Neuroanatomical studies in adults are more numerous, but have yielded inconsistent results, perhaps due to small samples and focused region-of-interest analyses (for review, see Kable and Levy, 2015). For example, it has been reported that greater DD (more impulsive choice) is associated with reduced gray matter volume in lateral prefrontal cortex (Bjork et al., 2009), superior frontal gyrus (Schwartz et al., 2010), and putamen (Dombrovski et al., 2012; Cho et al., 2013). Furthermore, greater DD has been associated with larger volume of the ventral striatum and posterior cingulate cortex (PCC; Schwartz et al., 2010), medial prefrontal regions and anterior cingulate cortex (ACC; Cho et al., 2013), as well as prefrontal cortex (Wang et al., 2016). One study of cortical thickness (CT) in adults revealed an association between higher DD and decreased CT in both medial prefrontal cortex and the ACC (Bernhardt et al., 2014). To our knowledge, there have been no neuroanatomical studies in adolescents that specifically examine the relationship between DD and CT. Notably, findings from adults may not necessarily extend to adolescents, given the dynamic remodeling of brain structure that occurs during this critical period (Sowell et al., 2004).

Accordingly, here we investigated how individual differences in DD may be associated with differences in brain structure during adolescence. To do this, we capitalized on a large sample of 427 youths imaged as part of the Philadelphia Neurodevelopmental Cohort (Satterthwaite et al., 2014a, 2016). We delineated covariance networks of CT using a recently developed application of non-negative matrix factorization for the multivariate analysis of high-dimensional neuroimaging data (Sotiras et al., 2015, 2017). We evaluated the association between DD and CT in each network, while specifically modeling both linear and nonlinear developmental effects using penalized splines. We hypothesized that we would find associations between DD and CT in brain regions associated with reward processing, such as the ventromedial prefrontal cortex (vmPFC; Kable and Glimcher, 2007; Bartra et al., 2013), as well as regions subserving cognitive control (e.g., dlPFC). As described below, diminished CT in these as well as other networks was associated with impulsive choice, and predicted individual variation in DD above and beyond that explained by cognitive performance.

\section{Materials and Methods}

Participants and sample construction. Participants were a subsample of 1601 youths recruited as part of the Philadelphia Neurodevelopmental Cohort (PNC) who underwent neurocognitive assessment (Gur et al., 2010, 2012), as well as neuroimaging (Satterthwaite et al., 2014a, 2016). A subsample of PNC participants $(n=453)$ completed the DD task. Of those, $n=2$ did not pass the quality control criteria for the task (described in the next subsection). Additionally, $n=24$ participants were excluded for the following reasons: health conditions that could impact brain structure $(n=19)$, scanning performed $>12$ months from DD testing $(n=1)$, inadequate structural image quality $(n=3)$, or missing imaging data $(n=1)$. The remaining $n=427$ participants constituted our final sample for analysis (mean age at scanning: $17.0 \pm 3.2$ years, age range: $9.3-24.3$ years; $48.7 \%, n=208$ males).

Delay discounting task. The DD task consisted of 34 self-paced questions where the participant chose between a smaller amount of money available immediately or a larger amount available after a delay. This task was modeled after the work of Senecal et al. (2012). The smaller, immediate rewards ranged between $\$ 10$ and $\$ 34$ and were always displayed at the top of the computer screen. The larger, delayed rewards were fixed at $\$ 25, \$ 30$, or $\$ 35$, with the delays ranging between 1 and $171 \mathrm{~d}$. Larger, delayed rewards were always displayed on the bottom of the screen. All rewards were hypothetical but participants were instructed to make decisions as if the choices were real. Discount rates based on hypothetical choices have shown no systematic differences from discount rates based on real rewards, in the same participants (Johnson and Bickel, 2002). The set of choices was identical in content and order for all participants. The DD task was administered as part of an hour-long web-based battery of neurocognitive tests (see below, Computerized Neurocognitive Battery), on a separate day from the imaging session. The mean interval between the DD task and imaging was 0.44 months with a SD of 1 month (range $0-8$ months).

Discount rates from the delay discounting task were calculated assuming a hyperbolic discounting model of the form: $S V=A /(1+k D)$, where $S V$ is the subjective value of the delayed reward, $A$ is amount of the delayed reward, $D$ is the delay in days, and $k$ is the subject-specific discount rate (Mazur, 1987). We used the 'fmincon' optimization algorithm in MATLAB (MathWorks; RRID:SCR_001622) to estimate the bestfitting $k$ from each participant's choice data. A higher $k$ value indicates steeper discounting of delayed rewards and thus more impulsive choices. As the distribution of discount rates is highly right-skewed, we used $\log$-transformed $k(\log k)$ in all analyses.

We performed quality control to ensure that participants were not responding randomly, and verified that their responses were a function of task variables which should be relevant to the choice. Although a hyperbolic discounting model has been shown to fit discounting data better than an exponential model (Kirby and Maraković, 1995), quality control was performed independently of assumptions about the shape of the discount function. Specifically, each participant's responses were fit using a logistic regression model, with predictors including the immediate amount, delayed amount, delay, their respective squared terms, and two-way interaction terms. We assessed goodness of fit of this model using the coefficient of discrimination (Tjur, 2009), and discarded DD data from any participant who had a value of $<0.20$.

Neurocognitive battery. Cognition was assessed using the University of Pennsylvania Computerized Neurocognitive Battery (Penn CNB; Gur et al., 2010, 2012) during the same session that delay discounting was evaluated. Briefly, this hour-long battery consisted of 14 tests administered in a fixed order, evaluating aspects of cognition, including executive control, episodic memory, complex reasoning, social cognition, and sensorimotor and motor speed. Except for the motor tests that only measure speed, each test provides measures of both accuracy and speed. Performance on the tests for each domain is summarized as cognitive factors obtained with exploratory factor analysis with an oblique rotation (Moore et al., 2015). Prior work has demonstrated that accuracy on this battery can be parsimoniously summarized as either one overall cognitive performance factor or three domain-specific factors, including executive function and complex reasoning combined, social cognition, and 
episodic memory (Moore et al., 2015). Associations between DD and factor scores for each of these dimensions were analyzed, as described the Experimental design and statistical analysis subsection.

Image acquisition and quality assurance. Image acquisition and processing were reported in detail previously (Satterthwaite et al., 2014a, 2016). Briefly, all data were acquired on a single scanner (Siemens TIM Trio 3 tesla 32-channel head coil) using the same imaging sequences for all participants. Structural brain scanning was completed using a magnetization-prepared, rapid acquisition gradient-echo (MPRAGE) T1-weighted image with the following parameters: TR $1810 \mathrm{~ms}$; TE 3.51 ms; FOV $180 \times 240 \mathrm{~mm}$; matrix $192 \times 256 ; 160$ slices; slice thickness/gap $1 / 0 \mathrm{~mm}$; TI $1100 \mathrm{~ms}$; flip angle $9^{\circ}$; effective voxel resolution of $0.93 \times$ $0.93 \times 1.00 \mathrm{~mm}$; total acquisition time 3:28 min.

Image quality assurance. T1 image quality was independently assessed by three expert image analysts; for full details of this procedure see Rosen et al. (2017). Briefly, before rating images, all three raters were trained to $>85 \%$ concordance with faculty consensus rating on an independent training sample of 100 images. Each rater evaluated every raw T1 image on a $0-2$ Likert scale, where unusable images were coded as " 0 ", usable images with some artifact were coded as " 1 ", and images with none or almost no artifact were coded as " 2 ". All images with an average rating of 0 were excluded from analyses $(n=3)$; of the remaining images in the final sample $n=2$ had an average manual rating of $0.67, n=16$ were rated as $1, n=16$ were rated as $1.33, n=35$ were rated as 1.67 , and the remaining $n=358$ had an average rating of 2 . As described in the Experimental design and statistical analysis subsection, these average manual quality ratings were included in sensitivity analyses. In addition, we examined the distribution of CT values within anatomically defined regions created using a multi-atlas labeling technique. For each region, we created a distribution of thickness values; subjects with a CT value $>2 \mathrm{SD}$ from the mean were flagged for that region. This procedure was repeated for all 98 cortical regions, and the number of flags was summed across regions; this summarized the number of regions per subject that had an outlying value. Subjects with $>2.5$ SD number of regional outliers were flagged for manual re-evaluation. Notably, this extensive postprocessing QA procedure did initially identify one subject who failed the Advanced Normalization Tools (ANTs) CT procedure. For this subject, minor parameter adjustments were made and the procedure was rerun, resulting in no subjects with major processing errors that required exclusion. Beyond this participant, there was no other manual intervention into standardized image processing procedures.

Image processing and CT estimation. Structural image processing for estimating CT used tools included in ANTs (Tustison et al., 2014; RRID: SCR_004757). To avoid registration bias and maximize sensitivity to detect regional effects that can be impacted by registration error, a custom adolescent template and tissue priors were created. Structural images were then processed and registered to this template using the ANTs CT pipeline (Tustison et al., 2014). This procedure includes brain extraction, N4 bias field correction (Tustison et al., 2010), Atropos probabilistic tissue segmentation (Avants et al., 2011a), the top-performing SyN diffeomorphic registration method (Klein et al., 2010; Avants et al., 2011b; RRID:SCR_004757), and direct estimation of CT in volumetric space (Das et al., 2009). Large-scale evaluation studies have shown that this highly accurate procedure for estimating CT is more sensitive to individual differences over the lifespan than comparable techniques (Tustison et al., 2014). CT images were downsampled to $2 \mathrm{~mm}$ voxels before applying non-negative matrix factorization, but no additional smoothing was performed.

Non-negative matrix factorization. Cortical thickness was estimated as described in the previous paragraph over the entire cortical surface. We sought to reduce CT in our sample into fewer dimensions, for two reasons. First, an efficient summary of CT data would allow us to evaluate only a small number of associations, rather than conduct voxelwise inference that may be vulnerable to substantial type I error (Eklund et al., 2016). Second, and importantly, prior work has shown that there are inherent patterns of covariance in CT (Zielinski et al., 2010; AlexanderBloch et al., 2013; Sotiras et al., 2015, 2017), and analyzing the data according to this covariance structure may enhance interpretability.

Accordingly, we achieved both goals by using non-negative matrix factorization (NMF) to identify structural networks where CT covaries consistently across individuals and brain regions (Sotiras et al., 2015). NMF has previously been shown to yield more interpretable and reproducible components than other decomposition techniques such as principal component analysis or independent component analysis (Sotiras et al., 2015, 2017). In contrast to the other techniques, NMF only yields compact networks with positive weights, which facilitates interpretation of effects.

The NMF algorithm takes as input a matrix $X$ containing voxelwise $C T$ estimates (dimensions: 128,155 voxels $\times 427$ participants), and approximates that matrix as a product of two matrices with non-negative elements: $X \cong B C$ (Fig. 1). The first matrix, $B$, is of size $V \times K$ and contains the estimated non-negative networks and their respective loadings on each of the $V$ voxels, where $K$ is the user-specified number of networks. The $B$ matrix ("CT loadings") is composed of coefficients that denote the relative contribution of each voxel to a given network. These nonnegative coefficients of the decomposition by necessity represent the entirety of the brain as a subject-specific addition of various parts. The second matrix, $C$, is of size $K \times N$ and contains subject-specific scores for each network. These subject-specific scores ("CT network scores") indicate the contribution of each network in reconstructing the original CT map for each individual, and were evaluated for associations with DD as described below. We examined multiple NMF solutions ranging from 2 to 30 networks (in steps of 2) and calculated reconstruction error for each solution as the Frobenius norm between the CT data matrix and its NMF approximation (Sotiras et al., 2015, 2017). The optimal number of components was chosen based on the elbow of the gradient of the reconstruction error, such that the solution is adequate to model the structure of the data without modeling random noise (Sotiras et al., 2017). Network loadings were visualized on the inflated populationaverage, landmark-, and surface-based cortical surfaces (Van Essen, 2005; RRID:SCR_002099) using Caret software (Van Essen et al., 2001; RRID:SCR_006260).

Regional parcellation using multi-atlas segmentation. To demonstrate that our results are robust to methodological variation, we also derived $\mathrm{CT}$ estimates in anatomically defined regions of interest. We used a topperforming multi-atlas labeling approach to parcellate the brain into anatomical regions. This procedure has proven advantages over standard single-atlas approaches and has won open analysis challenges (Wang et al., 2013). Specifically, we used 24 young adult T1 images from the OASIS dataset (Marcus et al., 2007; RRID:SCR_007385), which have been manually labeled by Neuromorphometrics (RRID:SCR_005656). These images were each registered to each participant's T1 image again using the top-performing SyN diffeomorphic registration method included in ANTs (Klein et al., 2010; Avants et al., 2011b; RRID:SCR_004757). Finally, a joint label fusion algorithm was used to synthesize the multiple warped atlas-labeled images into a final segmentation consisting of 98 gray matter regions (Wang et al., 2013). Mean thickness was calculated within each of these regions, and evaluated in group-level analyses identical to those conducted for NMF-derived networks, as described in the next subsection.

Experimental design and statistical analysis. To examine associations between DD and brain structure, we used a cross-sectional sample of youths recruited as part of a large neurodevelopmental study. Brain development is frequently a nonlinear process (Giedd et al., 1999; Lenroot et al., 2007; Satterthwaite et al., 2014b). To capture both linear and nonlinear age effects, we modeled age with a penalized spline within generalized additive models (GAMs; Wood, 2004, 2011; Vandekar et al., 2015). In this type of model, a penalty is assessed on nonlinearity using restricted maximum likelihood to avoid overfitting. GAMs were implemented in the R package mgcv RRID:SCR_001905.

GAMs were first used to test for associations between DD and demographic variables such as age and sex. Next, we evaluated the association between DD and cognitive performance (as summarized by the overall cognitive performance factor and three domain-specific factor scores described above), while covarying for sex and age. In both sets of analyses, DD was used as the dependent variable. Finally, univariate associations between DD and NMF-derived structural covariance networks were evaluated, with CT scores as the dependent variables and controlling for sex and age. Interactions between DD and age were evaluated but 


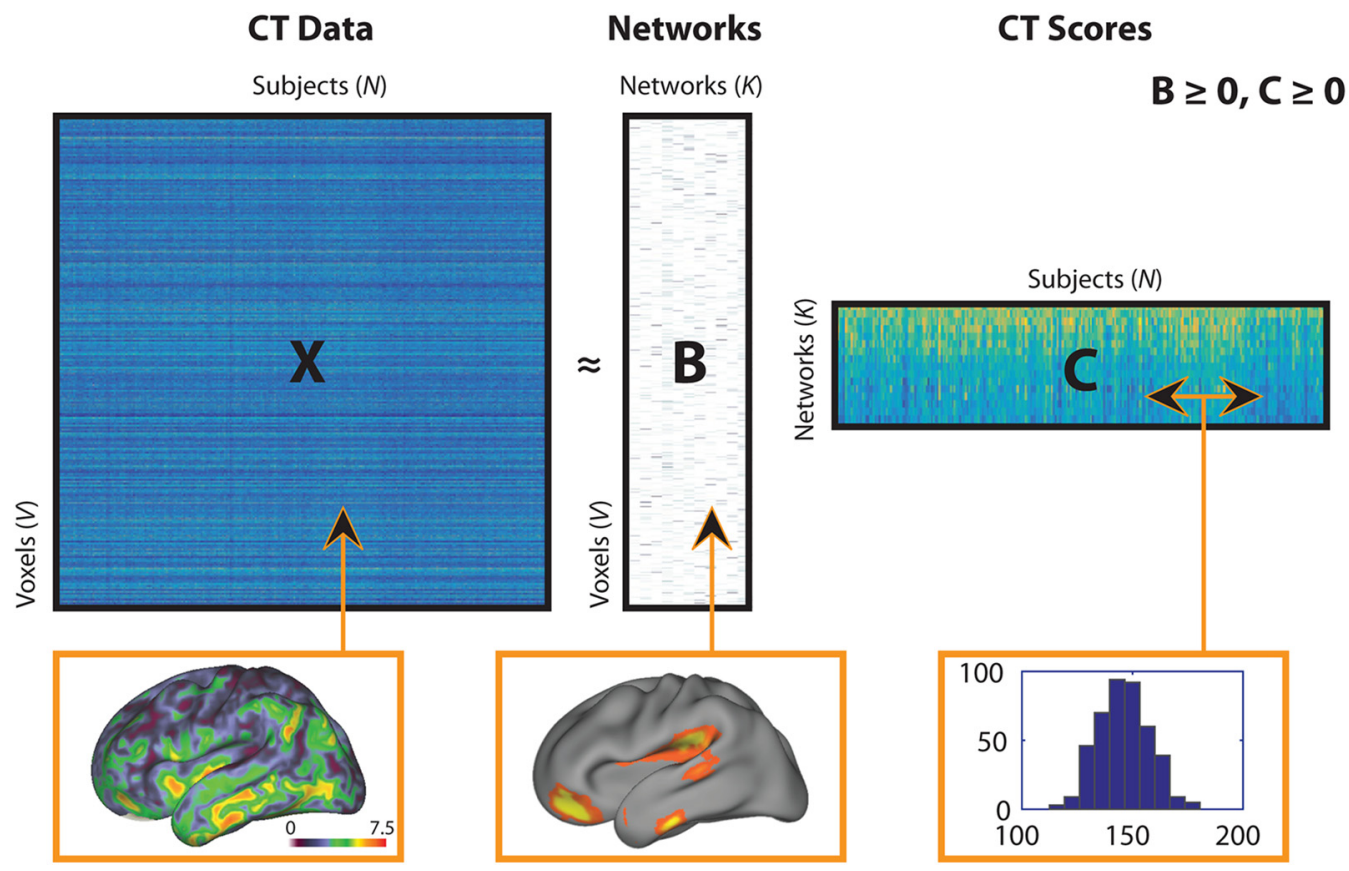

Figure 1. Schematic of non-negative matrix factorization and example data for each matrix. The $X$ matrix represents the CT data (columns) for all subjects (rows); the CT map shows example CT data from one participant, and corresponds to a column in the $X$ matrix. The $B$ matrix represents estimated networks (columns) and their loadings on each voxel (rows); the example map shows loadings from one network, and corresponds to a column in the $B$ matrix. The ( matrix provides the subject-specific weights (columns) for each network (rows); the histogram shows $C T$ scores in a single network, and corresponds to a row in the $($ matrix. Matrix sizes are shown with following dimensions: $V=$ number of $C T$ voxels, $N=$ number of participants; $K=$ number of networks.

were not found to be significant, and were thus not included in the univariate models. To control for multiple testing across either cognitive factors or structural covariance networks, we used the false discovery rate (FDR; $Q<0.05$; Benjamini and Hochberg, 1995).

To ensure that our results were not driven by potentially confounding factors, we conducted several sensitivity analyses. First, to ensure that our results were not driven by socioeconomic status (SES), nonspecific neurostructural effects, data quality, or general cognitive abilities, we repeated these analyses while including maternal education, total brain volume, mean image quality rating, and the overall cognitive performance factor as model covariates in separate models. Second, we repeated our analyses while excluding participants who were taking a psychotropic medication at the time of scan $(n=52)$ or for whom medication data were not available $(n=3)$ to ensure that these participants did not bias the observed results.

Multivariate analyses. The analyses described in the previous subsection examined univariate associations between each structural covariance network and DD. As a final step, we also investigated the multivariate predictive power of all cortical networks considered simultaneously, over and above that of two reduced models that included only demographics and non-neural correlates of DD (specifically, cognitive performance or maternal education). The first full model predicted DD using all 19 NMF networks, as well as age, sex, and the cognitive factors that were significantly associated with DD. The second full model predicted DD using all 19 NMF networks, as well as demographic variables including age, sex, and maternal education. These full models were compared with the reduced models (without the CT networks) using $F$ tests.

\section{Results}

\section{Impulsive choice is associated with reduced} cognitive performance

Mean discount rate in our sample was $0.073 \pm 0.088$. Delay discounting was not related to demographic variables including age $(p=0.387)$. There was a nonsignificant trend toward more impulsive discounting in males $(p=0.07)$, and this trend was most prominent at younger ages (age by sex interaction: $p=0.09$ ). In contrast, delay discounting was significantly associated with cog- nitive performance: youth who had higher discount rates also tended to have lower overall cognitive performance (partial $r=$ $-0.26, p<0.0001)$. Follow-up analyses with a three-factor model describing specific cognitive domains revealed that this effect was driven primarily by an association with a combined executive functioning and complex reasoning factor (partial $r=$ $-0.29, p<0.0001)$. Greater discounting was also associated with diminished memory accuracy (partial $r=-0.20, p<0.0001$ ), whereas there was no significant relationship between DD and social cognition (partial $r=-0.08, p=0.10$ ).

\section{Non-negative matrix factorization identifies structural covariance networks}

Next, we sought to identify structural covariance networks in CT using NMF. NMF provides a data-driven way to identify structural covariance networks, where CT varies in a consistent way across individuals. As NMF identifies structural networks at a resolution set by the user, we examined solutions ranging from 2 to 30 networks (in steps of 2). As expected, reconstruction error consistently decreased as the number of networks increased. Similar to previous applications of this method (Sotiras et al., 2015), reconstruction error stabilized at 20 networks (Fig. 2). Accordingly, the 20-network solution was used for all subsequent analyses (Fig. 3).

As in prior work using NMF (Sotiras et al., 2017), the structural covariance networks identified were highly symmetric bilaterally. Networks included specific cortical regions that are relevant to reward processing and decision-making, such as vmPFC and orbitofrontal cortex (OFC). Notably, when combined, several of the networks corresponded to aspects of functional brain networks. For example, networks 1 and 3 loaded on ACC and anterior insula, respectively, similar to the "salience network" (Seeley et al., 2007). Furthermore, specific networks defined lower-order systems, including motor (network 11) and 


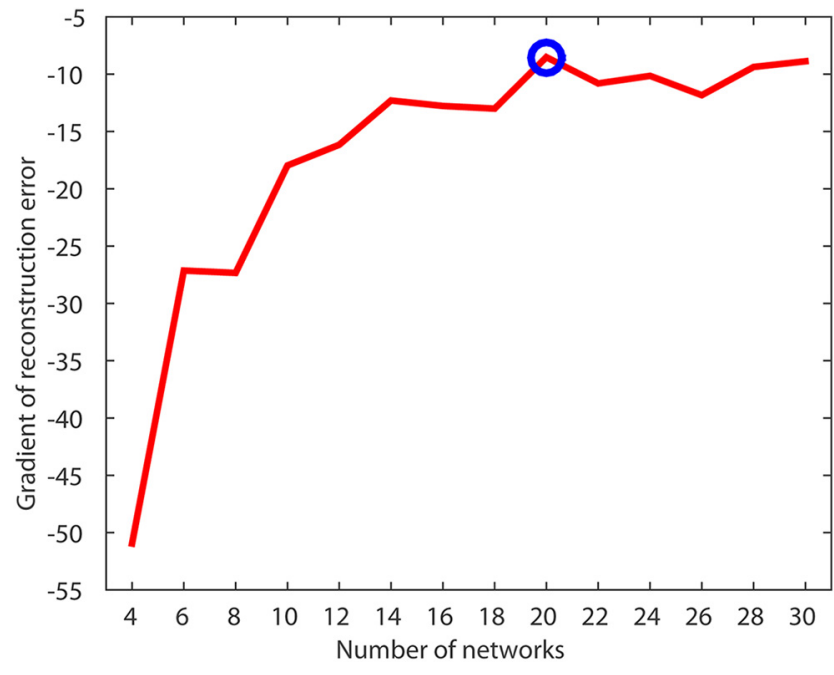

Figure 2. NMF reconstruction error identifies 20 cortical networks as the optimal resolution for CT data. Plot of reconstruction error gradient for NMF at multiple resolutions; the gradient is the difference in reconstruction error as the NMF solution increases by two networks. Blue circle indicates selected NMF solution of 20 networks.

visual (network 12) cortex. The 20-network solution also included a noise component (network 17), which was subsequently excluded from all analyses, resulting in 19 networks evaluated in total.

\section{Greater delay discounting is associated with diminished CT}

Having identified 19 interpretable structural covariance networks using NMF, we next examined associations with DD while controlling for sex as well as linear and nonlinear age effects using penalized splines. Univariate analyses revealed that there was a significant association (after FDR correction) in 11 networks (Table 1). In each of these networks, impulsive choice, indicated by high discount rates, was associated with diminished CT. Notably, the strongest effects were found in two networks comprised of the ventromedial prefrontal cortex and orbitofrontal cortex, both regions known to be critical for reward-related decisionmaking. These two networks also included parts of the temporal pole and temporoparietal junction (TPJ; networks 14 and 15; Fig. 4). Other networks where DD was associated with reduced CT included the temporal poles (network 9), lateral (network 8) and posterior temporal (network 20) lobes, dorsolateral prefrontal cortex (network 18), insula (network 3), fusiform gyrus (network 7 ), frontoparietal cortex (network 11), and visual cortex (network 12).

Association between CT and delay discounting is independent of age-related changes in CT

Having established that individual differences in DD are associated with CT, we next examined whether this effect was moderated by age. Notably, there was no significant age by DD interaction in any network (median $p=0.77$, range: $0.09-0.94$ ). Thus, age-related changes in CT were similar in both high and low discounters, but those with higher discount rates had thinner cortex across the age range examined (Fig. 5).

\section{Sensitivity analyses provide convergent results}

We conducted sensitivity analyses to evaluate potentially confounding variables including maternal education, total brain volume, image data quality, general cognitive abilities, and psychotropic medications. First, we examined whether the results could be explained by differences in maternal education, a proxy of socioeconomic status. Discount rate was significantly associated with maternal education (partial $r=-0.164, p=0.0007$ ), but including it in the model did not have a great impact on results. Specifically, 7 of 11 networks found to be related to DD remained FDR-significant, including the vmPFC and OFC networks; the other 4 networks trended toward significance $\left(p_{\mathrm{fdr}}<\right.$ 0.067). Second, we examined the effect of total brain volume on our findings. After adding total brain volume as a covariate, 10 of 11 networks remained FDR-significant for association with DD, with the remaining network showing a trend toward FDRsignificance $\left(p_{\mathrm{fdr}}=0.0762\right)$. Third, we included mean image quality rating (averaged across three expert raters) as a model covariate. Despite the fact that data quality was significantly related to discount rate (Spearman's partial $\rho=-0.159, p=$ 0.001), 7 of 11 networks continued to have an FDR-significant association after inclusion of this covariate (including the vmPFC and OFC networks), and 3 of 11 networks had FDR-corrected $p$ values $<0.10$. Fourth, we examined the effect of cognitive abilities, as measured by the overall cognitive performance factor. After including this variable as a covariate, 5 of 11 networks related to DD remained FDR-significant, including networks spanning the vmPFC, OFC, insula, and inferior temporal lobe. Finally, we repeated this analysis after excluding 52 participants who were taking psychotropic medication at the time of scan and 3 participants for whom medication data were missing. Despite the reduced power of this smaller sample, 10 of 11 networks remained FDR-significant, with the final network showing a trend toward significance $\left(p_{\mathrm{fdr}}=0.0503\right)$.

\section{Analyses with anatomically defined regions yield similar results}

To evaluate the robustness of the relationship between DD and CT to methodological variation, we also examined associations within 98 anatomically based regions. Univariate analyses controlling for sex and age revealed significant negative associations between DD and CT in 24 of these regions (Fig. 6). Consistent with the previously described NMF results, impulsive choice, indexed by greater discounting, was associated with diminished CT in medial frontal cortex, OFC, fusiform gyrus, frontal and temporal poles, insular cortex, middle and superior temporal gyri, precentral gyrus, and occipital cortex.

\section{Covariance networks provide improved prediction of DD over demographic and cognitive data}

The univariate analyses described above demonstrated that reduced CT in several structural covariance networks is associated with impulsive choice. Next, we tested whether a multivariate model including all structural networks could accurately predict $\mathrm{DD}$ on an individual basis. Delay discounting predicted from a model of CT scores in all 19 networks, as well demographic data (age and sex), was significantly correlated with actual delay discounting behavior $(r=0.33, p<0.0001$; Fig. 7). Adding CT scores to a reduced model with demographics alone improved model fit $\left(F_{(405,424)}=2.37, p=0.001\right)$; DD predicted from this reduced model with demographics only achieved a correlation of 0.097 ( $p=0.043$ ) with actual $\log k$ values.

Importantly, CT data also improved prediction above and beyond that achieved by cognitive predictors: adding CT scores to a model with cognitive performance as well as demographics improved the model fit $\left(F_{(403,422)}=1.63, p=0.047\right)$. DD predicted from the reduced model with just demographics and cognition achieved a correlation of $0.31(p<0.0001)$ between 
model-predicted and actual $\log k$ values compared with a correlation of $0.40(p<$ $0.0001)$ from a complete model including CT data, cognitive data, and demographics. Furthermore, CT networks also improved prediction above and beyond that achieved by maternal education, a proxy of SES, which was correlated with DD $\left(F_{(401,420)}=1.97, p=0.009\right)$. DD predicted from a model with demographic variables such as age, sex, and maternal education achieved a correlation of 0.19 $(p<0.0001)$ between predicted and actual $k$ values, whereas $k$ values estimated from the full model with CT data, demographics, and maternal education achieved a correlation of $0.34(p<0.0001)$ with actual $k$ values.

\section{Discussion}

We examined associations between delay discounting and CT networks in a large adolescent sample. More impulsive preferences, as indexed by higher discounting, were associated with diminished CT in multiple networks. The strongest effects were found in OFC, vmPFC, temporal pole, and the TPJ. Associations between DD and brain structure did not vary over the age range studied, and could not be explained by confounding variables. Furthermore, consideration of structural networks improved prediction of DD above and beyond demographic and cognitive variables.

\section{Structural covariance networks related}

\section{to DD overlap with known functional networks}

Greater discounting was associated with decreased CT in multiple structural networks. Relative to previous reports of both neurofunctional and neurostructural correlates of DD (Peters and Büchel, 2011; Bernhardt et al., 2014; Kable and Levy, 2015), the effects we observed were fairly widespread across the brain. Notably, many of the regions encompassed by these networks correspond to findings from previous studies in adults, including functional networks known to be involved in DD. As hypothesized, we found associations between DD and CT in central elements of the valuation network, namely vmPFC (Bartra et al., 2013), the cognitive control network, including dlPFC (Peters and Büchel, 2011; Stanger et al., 2013), and the prospection network, involving medial temporal cortex (Peters and Büchel, 2011). Although DD and CT relationships have not previously been evaluated in adolescents, one prior study documented diminished thickness in the ACC and medial PFC in association with greater DD in adults (Bernhardt et al., 2014). In addition to hypothesized effects, we also found associations between DD and CT in motor, somatosensory, and both early and higher-order visual cortices. Notably, when these effects were evaluated jointly in a multivariate model, CT networks enhanced prediction of DD above and beyond demographic and cognitive variables. This result contributes to efforts in neuroeconomics to improve prediction of decision-making behavior using brain-based measures obtained independently of the behavior itself (Kable and Levy,
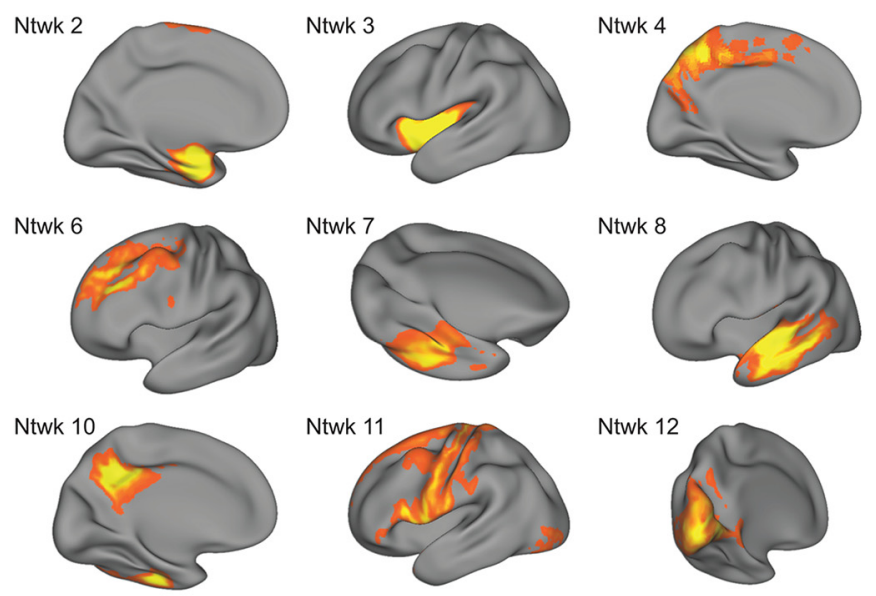

Ntwk 12
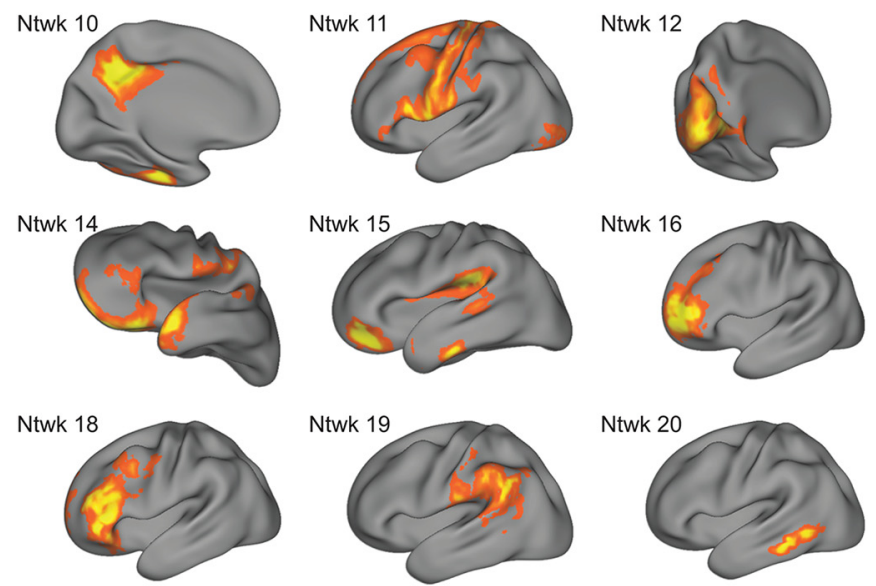

Figure 3. Structural covariance networks delineated by NMF. Visualization of structural covariance networks from the 20network NMF solution. The spatial distribution of each network is indicated by loadings at each voxel in arbitrary units (from $B$ ix in NMF factorization); warmer colors represent higher loadings. For each network, we show one view that best captures the

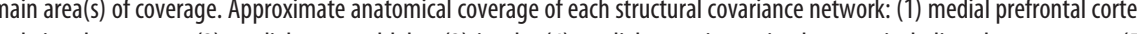
temporooccipital cortex; (6) dIPFC; (7) fusiform gyrus; (8) lateral temporal lobe; (9) lateral temporal lobe and temporal pole; (10) (12) (13) medial temporal cortex, ACC, and PCC; (14) OFC, frontal and temporal poles; (15) vmPFC, inferio temporal lobe, auditory cortex, and TPJ; (16) dorsal OFC; (17) the dura matter, a noise component that was not evaluated further; (18) dIPFC; (19) angular and supramarginal gyri; and (20) posterior inferior temporal lobe.

Table 1. Association between delay discounting and NMF-derived structural covariance networks

\begin{tabular}{|c|c|c|c|c|c|c|}
\hline Networks & $\beta$ & SE & $t$ & $p$ & $F D R-p$ & $r$ \\
\hline 1 & -0.649 & 0.3946 & -1.64 & 0.101 & 0.137 & -0.080 \\
\hline 2 & -0.0138 & 0.4217 & -0.03 & 0.974 & 0.974 & 0.002 \\
\hline 3 & -1.5868 & 0.5606 & -2.83 & 0.005 & 0.019 & -0.136 \\
\hline 4 & -0.4337 & 0.6414 & -0.68 & 0.499 & 0.527 & -0.033 \\
\hline 5 & -0.9959 & 0.4811 & -2.07 & 0.039 & 0.062 & -0.100 \\
\hline 6 & -0.8277 & 0.5337 & -1.55 & 0.122 & 0.154 & -0.075 \\
\hline 7 & -1.1428 & 0.4359 & -2.62 & 0.009 & 0.024 & -0.126 \\
\hline 8 & -1.1598 & 0.4562 & -2.54 & 0.011 & 0.024 & -0.123 \\
\hline 9 & -0.7926 & 0.3580 & -2.21 & 0.027 & 0.047 & -0.110 \\
\hline 10 & -0.3748 & 0.3055 & -1.23 & 0.221 & 0.262 & -0.060 \\
\hline 11 & -1.1527 & 0.4669 & -2.47 & 0.014 & 0.027 & -0.119 \\
\hline 12 & -1.5839 & 0.6164 & -2.57 & 0.011 & 0.024 & -0.124 \\
\hline 13 & -1.173 & 0.4283 & -2.74 & 0.006 & 0.02 & -0.132 \\
\hline 14 & -2.019 & 0.4241 & -4.76 & $<0.0001$ & $<0.0001$ & -0.225 \\
\hline 15 & -1.257 & 0.3036 & -4.14 & $<0.0001$ & $<0.0001$ & -0.200 \\
\hline 16 & -0.4404 & 0.4371 & -1.01 & 0.314 & 0.351 & -0.050 \\
\hline 18 & -1.252 & 0.4305 & -2.91 & 0.004 & 0.018 & -0.140 \\
\hline 19 & -0.7172 & 0.3713 & -1.93 & 0.054 & 0.079 & -0.094 \\
\hline 20 & -0.8778 & 0.3014 & -2.91 & 0.004 & 0.018 & -0.140 \\
\hline
\end{tabular}

$\beta$ (unstandardized regression coefficient), SE ( $\beta$ 's SE), $t$ ( $t$ value for testing $\beta$ against 0 ; dfs $=423$ ), $p$ value, and FDR-corrected $p$ values are obtained from separate general additive models run for each network. In this model, discount rate (log $k$ ) predicts (T scores, controlling for age (fit as a penalized spline) and sex. As an estimate of the linear effect size, $r$ is the partial Pearson's correlation coefficient between discount rate and CT scores in each network while adjusting for linear age, quadratic age, and sex. 


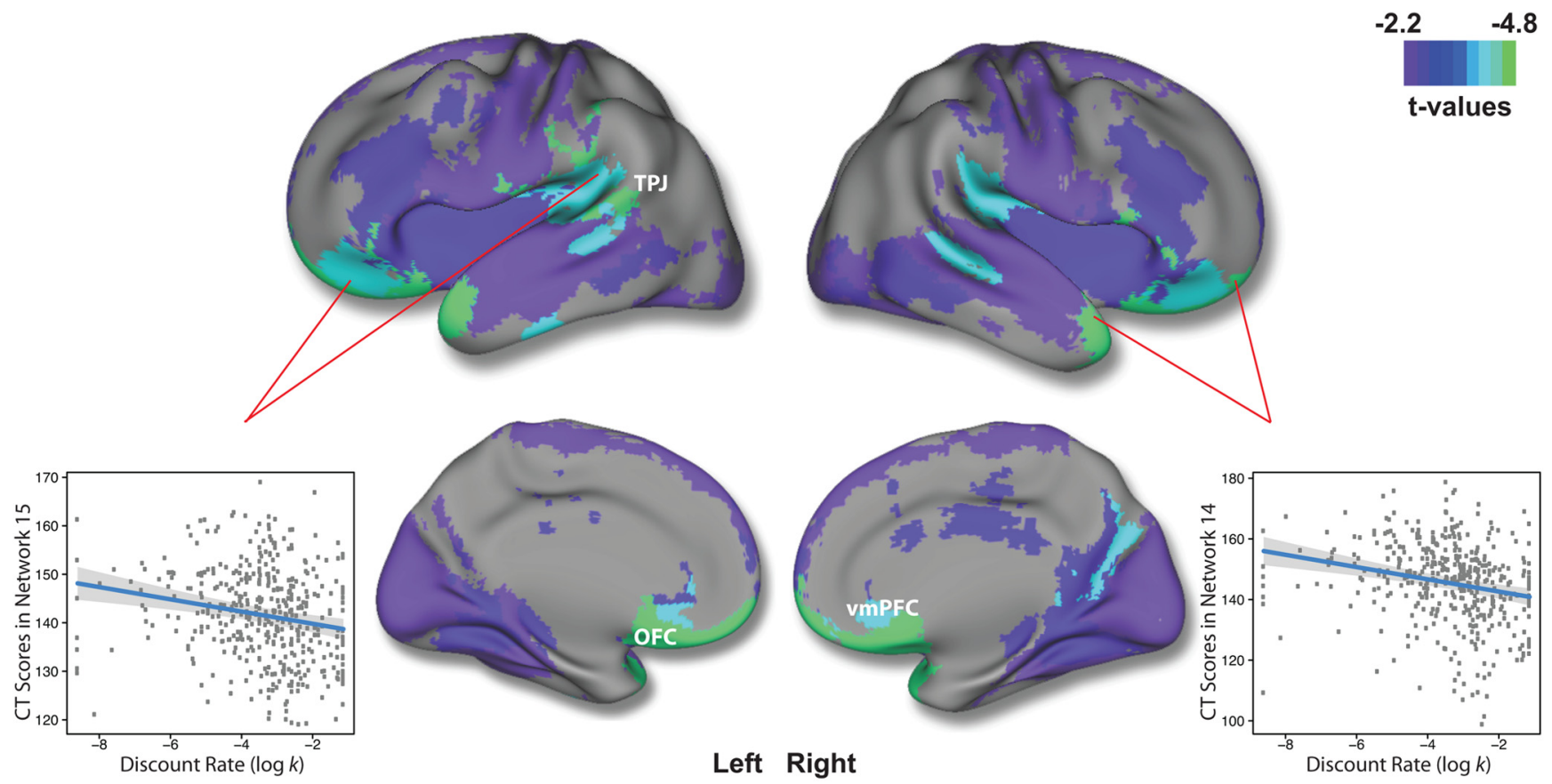

Figure 4. Higher discounting is associated with diminished CT in frontal, temporal, and parietal areas. Regions of FDR-significant association between log $k$ and structural covariance networks. The composite network visualization was obtained by assigning each voxel to the network which has the highest loading for that voxel (from the $B$ matrix), across all 19 networks. Maximal effects were observed in networks 14 and 15, which included orbitofrontal cortex and ventromedial prefrontal cortex. Scatterplots for log $k$-CT association in these networks are shown, while adjusting for model covariates. Gray envelope represents the $95 \%$ confidence interval.
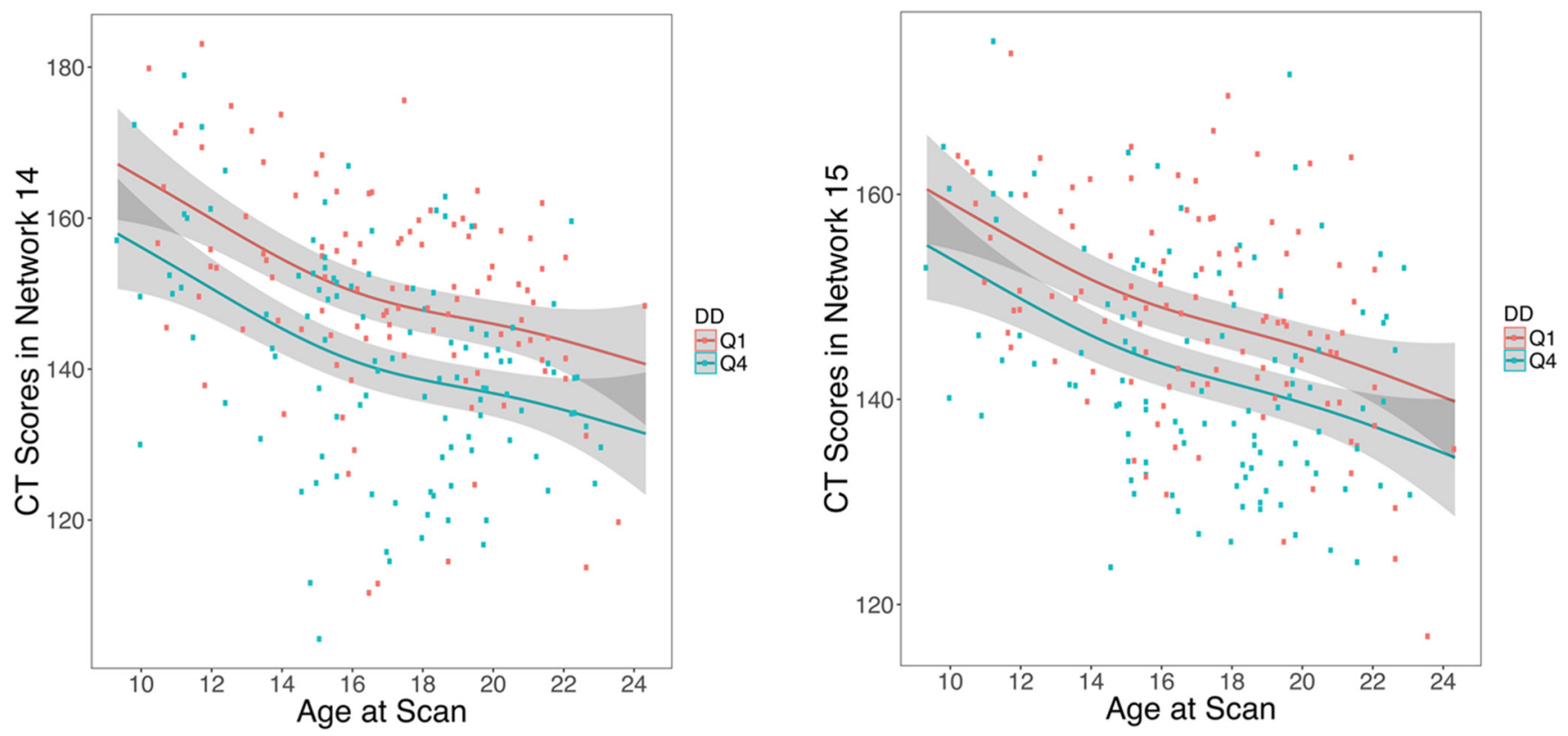

Figure 5. Association between CT and delay discounting is independent of age. Scatterplots for relationship between age and CT in networks 14 and 15 separated by top (Q4) and bottom ( 01 ) quartiles of $\log k$. The Q4 quartile group contains participants with the most impulsive preferences. For each quartile, the age-CT relationship is shown after adjusting for model covariates, and includes the $95 \%$ confidence intervals (gray envelopes).

2015), and suggests that structural covariance networks may be a useful marker of impulsive choice in youth.

\section{Results converge with data from lesion and neuromodulation studies}

Although the negative associations between DD and CT were widespread and distributed, two structural covariance networks exhibited particularly strong associations with DD. Brain regions comprising these networks included vmPFC, OFC, temporal pole, and the TPJ. As mentioned above, our findings in vmPFC were expected based on substantial evidence from fMRI studies that this region is implicated in DD (Kable and Glimcher, 2007; Ballard and Knutson, 2009; Bartra et al., 2013). Furthermore, activity in vmPFC when merely thinking about the future predicts DD, such that lower discounters show greater activity when thinking about the far future (Cooper et al., 2013). Finally, consistent with our results, a study in 


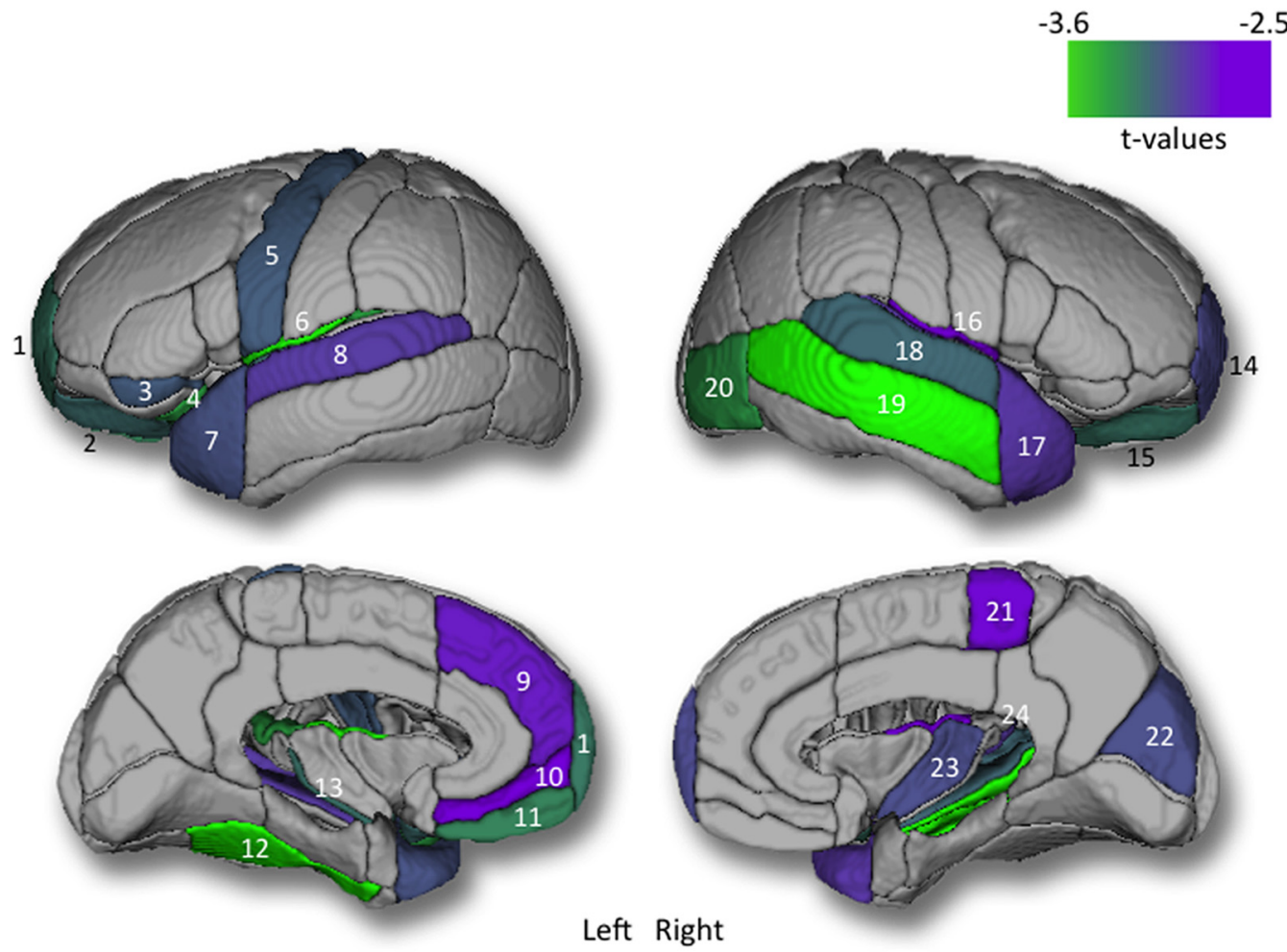

Figure 6. Higher discounting is associated with diminished regional CT in frontal and temporal regions. FDR-significant associations between log $k$ and $C T$ estimated in anatomically defined regions. Significant regions include the following: left frontal pole (1); left medial orbital gyrus (2); left orbital part of the inferior frontal gyrus (3); left posterior orbital gyrus (4); left precentral gyrus (5); left central operculum (6); left temporal pole (7); left superior temporal gyrus (8); left superior frontal gyrus, medial segment (9); left medial frontal cortex (10); left gyrus rectus (11); left fusiform gyrus (12); left planum polare (13); right frontal pole (14); right medial orbital gyrus (15); right central operculum (16); right temporal pole (17); right superior temporal gyrus (18); middle temporal gyrus (19); right inferior occipital gyrus (20); right precentral gyrus, medial segment (21); right cuneus (22); right posterior insula (23); and right planum temporale (24).

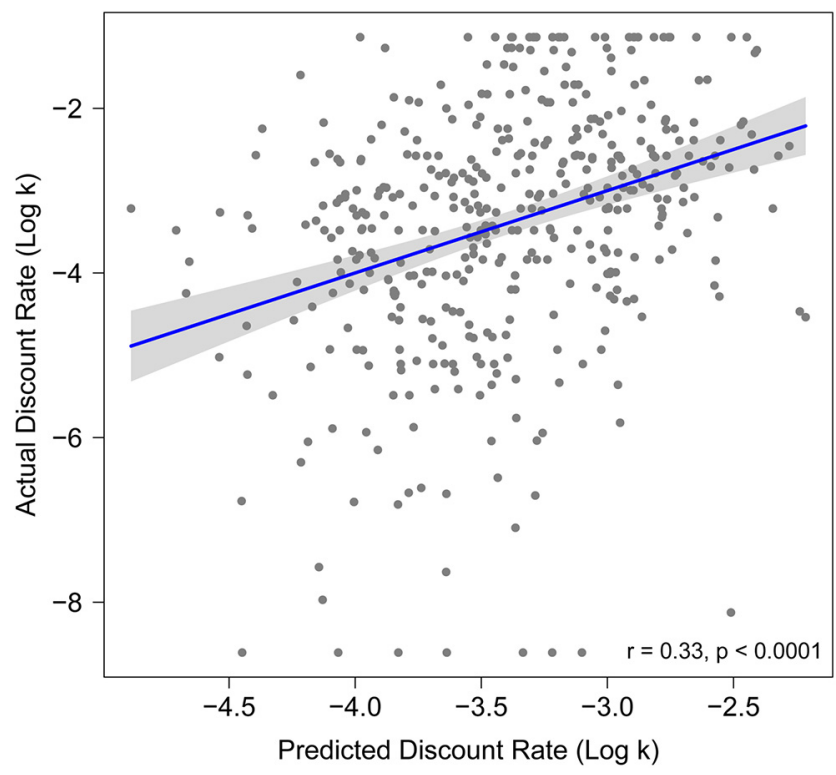

Figure 7. CT data from structural covariance networks predicts delay discounting. Scatterplot for relationship between actual $\log k$ values and predicted $\log k$ from multivariate CT prediction. Multivariate prediction is based on CT scores from all structural covariance networks plus demographic variables: sex and age. Scatterplots include line of best fit for this association with a $95 \%$ confidence interval (gray envelope).

adults reported that diminished $\mathrm{CT}$ in that region was associated with higher DD (Bernhardt et al., 2014).

Beyond the vmPFC, evidence suggests that regions including the OFC, temporal pole, and TPJ are both involved in and neces- sary for evaluating future outcomes in DD. First, lesion studies in patients with medial OFC damage show greater discounting of both primary and secondary rewards, compared with healthy controls and non-frontal damage patients (Sellitto et al., 2010), and this is the only region where injuries have been reported to increase discounting in humans. Notably, this relationship is dose-dependent, such that larger frontal lesions are associated with steeper discounting. Second, patients with semantic dementia, a disorder characterized by anterior temporal lobe atrophy, show greater discounting than controls (Chiong et al., 2016). Third, although the TPJ has typically been implicated in social cognition and theory of mind, recent data suggest that it also plays a role in both monetary and social discounting (Strombach et al., 2015; Soutschek et al., 2016). Importantly, disrupting the TPJ in healthy adults using transcranial magnetic stimulation increases discounting (Soutschek et al., 2016). Collectively, this evidence suggests that the disruption of OFC, anterior temporal lobe, and TPJ may promote impulsive choice.

\section{Associations with delay discounting are independent of age-related changes}

Although we replicated prior findings of association between lower discounting and better cognitive performance (Shamosh and Gray, 2008; Shamosh et al., 2008), we did not find significant associations between DD and age (Scheres et al., 2006; Steinberg et al., 2009). This may be due to differences in sample composition, including relatively less dense sampling of younger ages and use of a dimensional rather than a stratified design that compared older and younger age groups. However, the lack of observed age effect is consistent with a recent review noting that age effects on 
DD are inconsistent and of a relatively small effect size (Romer et al., 2017). Notably, the association between brain structure and DD was stable across the entire age range surveyed in our sample. This result is consistent with a prior study of DD in adolescents and white matter integrity assessed using diffusion imaging (Olson et al., 2009). Together, these results imply that individual differences in brain structure associated with impulsive choice do not emerge specifically during adolescence. These results may also suggest that such individual differences in brain structure may emerge early in development, consistent with literature describing the importance of structural brain development in utero, during the perinatal period, and during early childhood (Thomason et al., 2013; Di Martino et al., 2014). Although speculative, future research may reveal that individual differences in brain structure which emerge early in life may impact evolving patterns of value and cognitive control system function in adolescence such individual differences may, in turn, contribute to impulsivity during this critical period (Casey et al., 2008; Bjork et al., 2010).

\section{Advantages of evaluating structural covariance networks in a large sample}

The greater spatial extent of observed significant associations between brain structure and DD compared with prior results may be due to several aspects of our study. First, the large sample size afforded greater statistical power, and thus greater sensitivity, to detect effects in multiple networks. Although the effect sizes of these associations were small, research documenting inflation of effect sizes in small studies suggests that our results are more likely to be an accurate reflection of the true effect size than data from modest samples (Button et al., 2013). Second, structural covariance networks defined by NMF provided a parsimonious summary of the high-dimensional imaging data. In contrast to anatomic atlases based on sulcal folding patterns, NMF identifies structural networks based on patterns of covariance in the data itself. This concise summary of the data limited multiple comparisons: we only evaluated 19 networks in our analyses, in contrast to the hundreds of thousands of voxels typically surveyed in mass-univariate voxel-based morphometry studies. This allowed us to use a rigorous FDR correction for all comparisons, rather than cluster-based inference that may produce substantial type I error rates in many common implementations (Eklund et al., 2016).

\section{Limitations}

Certain limitations of this study should be noted. First, the observed effects were independent of age, suggesting that differences in brain structure associated with impulsive choice may emerge earlier than the examined age range. Future investigations should consider longitudinal designs including early childhood to precisely capture the emergence of these effects. Second, we used hypothetical instead of real rewards in the DD task. However, prior studies have yielded similar results in both behavioral (Johnson and Bickel, 2002) and functional neuroimaging paradigms (Bickel et al., 2009). Third, we cannot completely rule out potential confounding variables that may be correlated with DD. Previous studies have described associations between CT and SES in adolescence (Mackey et al., 2015), though importantly our results remained largely unaffected after controlling for maternal education, a proxy of SES. Fourth, although the ANTs DiReCT method of quantifying CT has been shown as highly accurate and more discriminative than comparable techniques in large-scale evaluation studies (Tustison et al., 2014), it does not allow high-resolution voxelwise analyses or provide information (such as surface area) regarding potentially important subcortical structures.

\section{Conclusions and future directions}

Understanding impulsive choice in adolescence is important because impulsivity is associated with a host of risky behaviors and outcomes, such as tobacco use (Reynolds, 2004), alcohol use (Fernie et al., 2013), obesity (Fields et al., 2013), and early sexual initiation (Khurana et al., 2012), which lead to substantial morbidity and mortality during adolescence. Leveraging a large developmental sample and advanced analytics, we found that individual variability in brain structure explains differences in DD in adolescence. Together, our results indicate that higher DD in youth is associated with reduced CT in multiple networks, including those known to be essential for valuation. These results emphasize that risky behaviors in adolescents should be considered in the context of individual differences of structural brain networks that are present early in life. Moving forward, such brain-based measures could potentially be used as biomarkers to identify youth at particularly high risk for negative outcomes. Future studies should evaluate associations between DD, brain structure, and psychopathology. Such efforts could potentially aid in stratifying youth within targeted clinical trials aiming to reduce impulsivity and risk-taking behaviors during this critical period.

\section{References}

Alexander-Bloch A, Giedd JN, Bullmore E (2013) Imaging structural covariance between human brain regions. Nat Rev Neurosci 14:322-336. CrossRef Medline

Audrain-McGovern J, Rodriguez D, Epstein LH, Cuevas J, Rodgers K, Wileyto EP (2009) Does delay discounting play an etiological role in smoking or is it a consequence of smoking? Drug Alcohol Depend 103: 99-106. CrossRef Medline

Avants BB, Tustison NJ, Wu J, Cook PA, Gee JC (2011a) An open source multivariate framework for $n$-tissue segmentation with evaluation on public data. Neuroinformatics 9:381-400. CrossRef Medline

Avants BB, Tustison NJ, Song G, Cook PA, Klein A, Gee JC (2011b) A reproducible evaluation of ANTs similarity metric performance in brain image registration. Neuroimage 54:2033-2044. CrossRef Medline

Ballard K, Knutson B (2009) Dissociable neural representations of future reward magnitude and delay during temporal discounting. Neuroimage 45:143-150. CrossRef Medline

Bartra O, McGuire JT, Kable JW (2013) The valuation system: a coordinatebased meta-analysis of BOLD fMRI experiments examining neural correlates of subjective value. Neuroimage 76:412-427. CrossRef Medline

Benjamini Y, Hochberg Y (1995) Controlling the false discovery rate: a practical and powerful approach to multiple testing. J Royal Stat Soc B 57:289-300.

Bernhardt BC, Smallwood J, Tusche A, Ruby FJ, Engen HG, Steinbeis N, Singer T (2014) Medial prefrontal and anterior cingulate cortical thickness predicts shared individual differences in self-generated thought and temporal discounting. Neuroimage 90:290-297. CrossRef Medline

Bickel WK, Miller ML, Yi R, Kowal BP, Lindquist DM, Pitcock JA (2007) Behavioral and neuroeconomics of drug addiction: competing neural systems and temporal discounting processes. Drug Alcohol Depend 90: S85-S91. CrossRef Medline

Bickel WK, Pitcock JA, Yi R, Angtuaco EJ (2009) Congruence of BOLD response across intertemporal choice conditions: fictive and real money gains and losses. J Neurosci 29:8839-8846. CrossRef Medline

Bjork JM, Momenan R, Hommer DW (2009) Delay discounting correlates with proportional lateral frontal cortex volumes. Biol Psychiatry 65:710 713. CrossRef Medline

Bjork JM, Smith AR, Chen G, Hommer DW (2010) Adolescents, adults and rewards: comparing motivational neurocircuitry recruitment using fMRI. PloS One 5:e11440. CrossRef Medline

Button KS, Ioannidis JP, Mokrysz C, Nosek BA, Flint J, Robinson ES, Munafò 
MR (2013) Power failure: why small sample size undermines the reliability of neuroscience. Nat Rev Neurosci 14:365-376. CrossRef Medline

Casey BJ, Jones RM, Hare TA (2008) The adolescent brain. Ann N Y Acad Sci 1124:111-126. CrossRef Medline

Chiong W, Wood KA, Beagle AJ, Hsu M, Kayser AS, Miller BL, Kramer JH (2016) Neuroeconomic dissociation of semantic dementia and behavioural variant frontotemporal dementia. Brain 139:578-587. CrossRef Medline

Cho SS, Pellecchia G, Aminian K, Ray N, Segura B, Obeso I, Strafella AP (2013) Morphometric correlation of impulsivity in medial prefrontal cortex. Brain Topogr 26:479-487. CrossRef Medline

Cooper N, Kable JW, Kim BK, Zauberman G (2013) Brain activity in valuation regions while thinking about the future predicts individual discount rates. J Neurosci 33:13150-13156. CrossRef Medline

Das SR, Avants BB, Grossman M, Gee JC (2009) Registration based cortical thickness measurement. Neuroimage 45:867-879. CrossRef Medline

Di Martino A, Fair DA, Kelly C, Satterthwaite TD, Castellanos FX, Thomason ME, Craddock RC, Luna B, Leventhal BL, Zuo XN, Milham MP (2014) Unraveling the miswired connectome: a developmental perspective. Neuron 83:1335-1353. CrossRef Medline

Dombrovski AY, Siegle GJ, Szanto K, Clark L, Reynolds CF, Aizenstein H (2012) The temptation of suicide: striatal gray matter, discounting of delayed rewards, and suicide attempts in late-life depression. Psychol Med 42:1203-1215. CrossRef Medline

Eaton DK, Kann L, Kinchen S, Shanklin S, Flint KH, Hawkins J, Harris WA, Lowry R, McManus T, Chyen D, Whittle L, Lim C, Wechsler H (2012) Youth risk behavior surveillance: United States, 2011. Morbidity and Mortality Weekly Report. Surveillance Summaries (Washington, DC: 2002) 61:1-162.

Eklund A, Nichols TE, Knutsson H (2016) Cluster failure: why fMRI inferences for spatial extent have inflated false-positive rates. Proc Natl Acad Sci U S A 113:7900-7905. CrossRef Medline

Fernie G, Peeters M, Gullo MJ, Christiansen P, Cole JC, Sumnall H, Field M (2013) Multiple behavioural impulsivity tasks predict prospective alcohol involvement in adolescents. Addiction 108:1916-1923. CrossRef Medline

Field M, Christiansen P, Cole J, Goudie A (2007) Delay discounting and the alcohol stroop in heavy drinking adolescents. Addiction 102:579-586. CrossRef Medline

Fields SA, Sabet M, Reynolds B (2013) Dimensions of impulsive behavior in obese, overweight, and healthy-weight adolescents. Appetite 70:60-66. CrossRef Medline

Giedd JN, Blumenthal J, Jeffries NO, Castellanos FX, Liu H, Zijdenbos A, Paus T, Evans AC, Rapoport JL (1999) Brain development during childhood and adolescence: a longitudinal MRI study. Nat Neurosci 2:861-863. CrossRef Medline

Gur RC, Richard J, Hughett P, Calkins ME, Macy L, Bilker WB, Brensinger C, Gur RE (2010) A cognitive neuroscience-based computerized battery for efficient measurement of individual differences: standardization and initial construct validation. J Neurosci Methods 187:254-262. CrossRef Medline

Gur RC, Richard J, Calkins ME, Chiavacci R, Hansen JA, Bilker WB, Loughead J, Connolly JJ, Qiu H, Mentch FD, Abou-Sleiman PM, Hakonarson H, Gur RE (2012) Age group and sex differences in performance on a computerized neurocognitive battery in children age 8-21. Neuropsychology 26:251-265. CrossRef Medline

Johnson MW, Bickel WK (2002) Within-subject comparison of real and hypothetical money rewards in delay discounting. J Exp Anal Behav 77: 129-146. CrossRef Medline

Kable JW (2013) Valuation, intertemporal choice, and self-control. In: Neuroeconomics: Decision making and the brain (Glimcher PW, Fehr E, eds), pp 173-192. Amsterdam: Elsevier.

Kable JW, Glimcher PW (2007) The neural correlates of subjective value during intertemporal choice. Nat Neurosci 10:1625-1633. CrossRef Medline

Kable JW, Levy I (2015) Neural markers of individual differences in decision-making. Curr Opin Behav Sci 5:100-107. CrossRef Medline

Khurana A, Romer D, Betancourt LM, Brodsky NL, Giannetta JM, Hurt H (2012) Early adolescent sexual debut: the mediating role of working memory ability, sensation seeking, and impulsivity. Dev Psychol 48: 1416-1428. CrossRef Medline

Kirby KN, Maraković NN (1995) Modeling myopic decisions: evidence for hyperbolic delay-discounting within subjects and amounts. Organ Behav Hum Dec Process 64:22-30. CrossRef

Klein A, Ghosh SS, Avants B, Yeo BT, Fischl B, Ardekani B, Gee JC, Mann JJ, Parsey RV (2010) Evaluation of volume-based and surface-based brain image registration methods. Neuroimage 51:214-220. CrossRef Medline

Lenroot RK, Gogtay N, Greenstein DK, Wells EM, Wallace GL, Clasen LS, Blumenthal JD, Lerch J, Zijdenbos AP, Evans AC, Thompson PM, Giedd JN (2007) Sexual dimorphism of brain developmental trajectories during childhood and adolescence. Neuroimage 36:1065-1073. CrossRef Medline

Mackey AP, Finn AS, Leonard JA, Jacoby-Senghor DS, West MR, Gabrieli CF, Gabrieli JD (2015) Neuroanatomical correlates of the incomeachievement gap. Psychol Sci 26:925-933. CrossRef Medline

Marcus DS, Wang TH, Parker J, Csernansky JG, Morris JC, Buckner RL (2007) Open access series of imaging studies (OASIS): cross-sectional MRI data in young, middle aged, nondemented, and demented older adults. J Cogn Neurosci 19:1498-1507. CrossRef Medline

Mazur JE (1987) An adjusting procedure for studying delayed reinforcement. In: Quantitative analyses of behavior, Vol. 5: the effect of delay and of intervening events on reinforcement value (Commons ML, Mazur JE, Nevin JA, eds) pp 55-73. Hillsdale, NJ: Lawrence Erlbaum.

Moore TM, Reise SP, Gur RE, Hakonarson H, Gur RC (2015) Psychometric properties of the penn computerized neurocognitive battery. Neuropsychology 29:235-246. CrossRef Medline

Olson EA, Collins PF, Hooper CJ, Muetzel R, Lim KO, Luciana M (2009) White matter integrity predicts delay discounting behavior in 9-to 23 year-olds: a diffusion tensor imaging study. J Cogn Neurosci 21:14061421. CrossRef Medline

Peters J, Büchel C (2011) The neural mechanisms of inter-temporal decision-making: understanding variability. Trends Cogn Sci 15:227-239. CrossRef Medline

Reynolds B (2004) Do high rates of cigarette consumption increase delay discounting? A cross-sectional comparison of adolescent smokers and young-adult smokers and nonsmokers. Behav Processes 67:545-549. CrossRef Medline

Romer D, Reyna VF, Satterthwaite TD (2017) Beyond stereotypes of adolescent risk taking: placing the adolescent brain in developmental context. Dev Cogn Neurosci 27:19-34. CrossRef Medline

Rosen A, Roalf DR, Ruparel K, Blake J, Seelaus K, Villa LP, Ciric R, Cook PA, Davatzikos C, Elliott MA, Garcia De La Garza A, Gennatas ED, Quarmley M, Schmitt JE, Shionhara RT, Tisdall MD, Craddock RC, Gur RE, Gur RC, Satterthwaite TD (2017) CrossRef Medline

Satterthwaite TD, Elliott MA, Ruparel K, Loughead J, Prabhakaran K, Calkins ME, Hopson R, Jackson C, Keefe J, Riley M, Mentch FD, Sleiman P, Verma R, Davatzikos C, Hakonarson H, Gur RC, Gur RE (2014a) Neuroimaging of the Philadelphia neurodevelopmental cohort. Neuroimage 86:544-553. CrossRef Medline

Satterthwaite TD, Shinohara RT, Wolf DH, Hopson RD, Elliott MA, Vandekar SN, Ruparel K, Calkins ME, Roalf DR, Gennatas ED, Jackson C, Erus G, Prabhakaran K, Davatzikos C, Detre JA, Hakonarson H, Gur RC, Gur RE (2014b) Impact of puberty on the evolution of cerebral perfusion during adolescence. Proc Natl Acad Sci U S A 111:8643-8648. CrossRef Medline

Satterthwaite TD, Connolly JJ, Ruparel K, Calkins ME, Jackson C, Elliott MA, Roalf DR, Ryan Hopsona KP, Behr M, Qiu H, Mentch FD, Chiavacci R, Sleiman PM, Gur RC, Hakonarson H, Gur RE (2016) The Philadelphia neurodevelopmental cohort: a publicly available resource for the study of normal and abnormal brain development in youth. Neuroimage 124: 1115-1119. CrossRef Medline

Scheres A, Dijkstra M, Ainslie E, Balkan J, Reynolds B, Sonuga-Barke E, Castellanos FX (2006) Temporal and probabilistic discounting of rewards in children and adolescents: effects of age and ADHD symptoms. Neuropsychologia 44:2092-2103. CrossRef Medline

Schwartz DL, Mitchell AD, Lahna DL, Luber HS, Huckans MS, Mitchell SH, Hoffman WF (2010) Global and local morphometric differences in recently abstinent methamphetamine-dependent individuals. Neuroimage 50:1392-1401. CrossRef Medline

Seeley WW, Menon V, Schatzberg AF, Keller J, Glover GH, Kenna H, Reiss AL, Greicius MD (2007) Dissociable intrinsic connectivity networks for salience processing and executive control. J Neurosci 27:2349-2356. CrossRef Medline

Sellitto M, Ciaramelli E, di Pellegrino G (2010) Myopic discounting of fu- 
ture rewards after medial orbitofrontal damage in humans. J Neurosci 30:16429-16436. CrossRef Medline

Senecal N, Wang T, Thompson E, Kable JW (2012) Normative arguments from experts and peers reduce delay discounting. Judgm Decis Mak 7:568-589. Medline

Shamosh NA, Gray JR (2008) Delay discounting and intelligence: a metaanalysis. Intelligence 36:289-305. CrossRef

Shamosh NA, DeYoung CG, Green AE, Reis DL, Johnson MR, Conway AR, Engle RW, Braver TS, Gray JR (2008) Individual differences in delay discounting: relation to intelligence, working memory, and anterior prefrontal cortex. Psychol Sci 19:904-911. CrossRef Medline

Sotiras A, Resnick SM, Davatzikos C (2015) Finding imaging patterns of structural covariance via non-negative matrix factorization. Neuroimage 108:1-16. CrossRef Medline

Sotiras A, Toledo JB, Gur RE, Gur RC, Satterthwaite TD, Davatzikos C (2017) Patterns of coordinated cortical remodeling during adolescence and their associations with functional specialization and evolutionary expansion. Proc Natl Acad Sci U S A 114:3527-3532. CrossRef Medline

Soutschek A, Ruff CC, Strombach T, Kalenscher T, Tobler PN (2016) Brain stimulation reveals crucial role of overcoming self-centeredness in selfcontrol. Sci Adv 2:e1600992. CrossRef Medline

Sowell ER, Thompson PM, Leonard CM, Welcome SE, Kan E, Toga AW (2004) Longitudinal mapping of cortical thickness and brain growth in normal children. J Neurosci 24:8223-8231. CrossRef Medline

Stanger C, Elton A, Ryan SR, James GA, Budney AJ, Kilts CD (2013) Neuroeconomics and adolescent substance abuse: individual differences in neural networks and delay discounting. J Am Acad Child Adolesc Psychiatry 52:747-755.e6. CrossRef Medline

Steinberg L, Graham S, O'Brien L, Woolard J, Cauffman E, Banich M (2009) Age differences in future orientation and delay discounting. Child Dev 80:28-44. CrossRef Medline

Strombach T, Weber B, Hangebrauk Z, Kenning P, Karipidis II, Tobler PN, Kalenscher T (2015) Social discounting involves modulation of neural value signals by temporoparietal junction. Proc Natl Acad Sci U S A 112: 1619-1624. CrossRef Medline

Thomason ME, Dassanayake MT, Shen S, Katkuri Y, Alexis M, Anderson AL, Yeo L, Mody S, Hernandez-Andrade E, Hassan SS, Studholme C, Jeong JW, Romero R (2013) Cross-hemispheric functional connectivity in the human fetal brain. Sci Transl Med 5:173ra24. CrossRef Medline
Tjur T (2009) Coefficients of determination in logistic regression models_a new proposal: the coefficient of discrimination. Am Stat 63:366372. CrossRef

Tustison NJ, Avants BB, Cook PA, Zheng Y, Egan A, Yushkevich PA, Gee JC (2010) N4ITK: improved N3 bias correction. IEEE Trans Med Imaging 29:1310-1320. CrossRef Medline

Tustison NJ, Cook PA, Klein A, Song G, Das SR, Duda JT, Kandel BM, van Strien N, Stone JR, Gee JC, Avants BB (2014) Large-scale evaluation of ANTs and FreeSurfer cortical thickness measurements. Neuroimage 99: 166-179. CrossRef Medline

Van Essen DC (2005) A population-average, landmark-and surface-based (PALS) atlas of human cerebral cortex. Neuroimage 28:635-662. CrossRef Medline

Van Essen DC, Drury HA, Dickson J, Harwell J, Hanlon D, Anderson CH (2001) An integrated software suite for surface-based analyses of cerebral cortex. J Am Med Inform Assoc 8:443-459. CrossRef Medline

Van Leijenhorst L, Gunther Moor B, Op de Macks ZA, Rombouts SA, Westenberg PM, Crone EA (2010) Adolescent risky decision-making: neurocognitive development of reward and control regions. Neuroimage 51: 345-355. CrossRef Medline

Vandekar SN, Shinohara RT, Raznahan A, Roalf DR, Ross M, DeLeo N, Ruparel K, Verma R, Wolf DH, Gur RC, Gur RE, Satterthwaite TD (2015) Topologically dissociable patterns of development of the human cerebral cortex. J Neurosci 35:599-609. CrossRef Medline

Wang H, Suh JW, Das SR, Pluta JB, Craige C, Yushkevich PA (2013) Multiatlas segmentation with joint label fusion. IEEE Trans Pattern Anal Mach Intell 35:611-623. CrossRef Medline

Wang Q, Chen C, Cai Y, Li S, Zhao X, Zheng L, Zhang H, Liu J, Chen C, Xue G (2016) Dissociated neural substrates underlying impulsive choice and impulsive action. Neuroimage 134:540-549. CrossRef Medline

Wood SN (2004) Stable and efficient multiple smoothing parameter estimation for generalized additive models. J Am Stat Assoc 99:673-686. CrossRef

Wood SN (2011) Fast stable restricted maximum likelihood and marginal likelihood estimation of semiparametric generalized linear models. J R Stat Soc Series B Stat Methodol 73:3-36. CrossRef

Zielinski BA, Gennatas ED, Zhou J, Seeley WW (2010) Network-level structural covariance in the developing brain. Proc Natl Acad Sci U S A 107: 18191-18196. CrossRef Medline 